\title{
Patients with colorectal cancer associated with Lynch syndrome and MLH1 promoter hypermethylation have similar prognoses
}

\author{
Sigurdis Haraldsdottir, MD, MS', Heather Hampel, MS, LGC², Christina Wu, MD³, Daniel Y. Weng, PhD \\ Peter G. Shields, MD³, Wendy L. Frankel, MD5${ }^{5}$, Xueliang Pan, $\mathrm{PhD}^{6}$, Albert de la Chapelle, MD, PhD ${ }^{2,4,7}$, \\ Richard M. Goldberg, MD³ and Tanios Bekaii-Saab, MD³
}

\begin{abstract}
Purpose: Mismatch repair-deficient (dMMR) colorectal cancer (CRC) is caused by Lynch syndrome (LS) in 3\% and sporadic inactivation of $M L H 1$ by hypermethylation $(M L H 1-\mathrm{hm})$ in $12 \%$ of cases. It is not clear whether outcomes between LS-associated and MLH1-hm CRC differ. The objective of this study was to explore differences in clinical factors and outcomes in these two groups.
\end{abstract}

Methods: Patients with dMMR CRC identified by immunohistochemistry staining and treated at a single institution from 1998 to 2012 were included. $M L H 1$-hm was established with BRAF mutational analysis or hypermethylation testing. Patients' charts were accessed for information on pathology, germ-line MMR mutation testing, and clinical course.

\section{INTRODUCTION}

Deficient mismatch repair (dMMR) activity is found in $15 \%$ of colorectal cancers (CRCs). Mismatch repair (MMR) genes remove errors in the form of deletions or insertions of DNA nucleotides that occur during the mitotic process. In the presence of dMMR activity, mismatched nucleotides are incorporated into cells, thus predisposing them to malignant transformation with a hypermutated phenotype. ${ }^{1}$ Microsatellite instability (MSI) is the hallmark of MMR deficiency. In 3\% of CRC cases, dMMR is caused by Lynch syndrome (LS) ${ }^{2}$ through germ-line mutations in the MLH1, MSH2, MSH6, PMS2, or $E P C A M^{3}$ genes, and in $12 \%$ of cases it is caused by sporadic inactivation of MLH1 (hypermethylation of the MLH1 gene promoter $(M L H 1-\mathrm{hm}))$. In rare cases it is caused by biallelic germ-line MMR mutations (constitutional MMR deficiency syndrome $)^{4}$; more recently, the biallelic occurrence of two
Results: A total of 143 patients had CRC associated with LS (37 patients, $26 \%$ ) or MLH1-hm (106 patients, 74\%). Patients with LS were younger, more often male, presented more often with stage III disease, and had more metachronous disease than patients with MLH1-hm tumors. There was no difference in cancer-specific survival (CSS) between the groups; overall survival was longer in patients with LS, but this difference was minimal after adjusting for age and stage at diagnosis.

Conclusion: CSS did not differ in LS-associated CRC compared with MLH1-hm CRC, suggesting that they carry a similar prognosis. Genet Med advance online publication 11 February 2016

Key Words: colorectal cancer; deficient mismatch repair system; hypermethylation; Lynch syndrome; survival

somatic MMR mutations were shown to explain some dMMR cases. $^{5,6}$

Tumors with dMMR have been associated with specific characteristics such as right-sided location, poor differentiation, lymphocytic infiltration, and mucinous features. ${ }^{7}$ They are also less likely to metastasize and tend to have a better overall survival in the early stages. ${ }^{8-10}$ Differences in clinical features and outcomes between LS-associated and MLH1-hm tumors have not been well explored, and many studies categorize the two subtypes without regard to their divergent origins as tumors with high MSI. Poynter et al. ${ }^{11}$ examined the incidence of MLH1-hm in CRC with high MSI in a population-based cohort and found hypermethylated tumors with high MSI to be significantly associated with older age, female gender, and a right-sided location when compared with nonmethylated tumors with high MSI. Tumor genomic studies suggest that

\footnotetext{
${ }^{1}$ Division of Medical Oncology, Department of Internal Medicine, Stanford University, Stanford, California, USA; ${ }^{2}$ Division of Human Genetics, Department of Internal Medicine, The Ohio State University Comprehensive Cancer Center, Arthur G. James Cancer Hospital and Richard J. Solove Research Institute, Columbus, Ohio, USA; ${ }^{3}$ Division of Medical Oncology, Department of Internal Medicine, The Ohio State University Comprehensive Cancer Center, Arthur G. James Cancer Hospital and Richard J. Solove Research Institute, Columbus, Ohio, USA; ${ }^{4}$ Comprehensive Cancer Center, The Ohio State University Comprehensive Cancer Center, Arthur G. James Cancer Hospital and Richard J. Solove Research Institute, Columbus, Ohio, USA; ${ }^{5}$ Department of Pathology, The Ohio State University Comprehensive Cancer Center, Arthur G. James Cancer Hospital and Richard J. Solove Research Institute, Columbus, Ohio, USA; ${ }^{6}$ Center for Biostatistics, The Ohio State University Comprehensive Cancer Center, Arthur G. James Cancer Hospital and Richard J. Solove Research Institute, Columbus, Ohio, USA; ${ }^{7}$ Department of Molecular Virology, Immunology and Medical Genetics, The Ohio State University Comprehensive Cancer Center, Arthur G. James Cancer Hospital and Richard J. Solove Research Institute, Columbus, Ohio, USA. Correspondence: Sigurdis Haraldsdottir (sharalds@stanford.edu)
} 
there is a molecular difference between tumors that develop in the setting of an inherited MMR mutation when compared with sporadic $M L H 1$-hm tumors, most notably with regard to the association of a BRAF mutation. BRAF mutations are observed in up to $60-70 \%$ of $M L H 1$-hm tumors but very rarely occur in LS-associated tumors. ${ }^{12,13}$

Universal immunohistochemistry (IHC) screening for MMR proteins in all CRC tumors was recommended by the Evaluation of Genomic Applications in Practice and Prevention workgroup of the Centers for Disease Control and Prevention in 2009 (ref. 12), by the National Comprehensive Cancer Network in March 2014 (ref. 14) and by the US Multi-Society Task Force in August 2014 (ref. 15). Data for MMR IHC in patients with CRC has been available at the Ohio State University since 1998 and has been performed routinely on all CRC tumors since 2006. The objective of this study was to retrospectively explore differences in clinical presentation and outcomes in patients with $\mathrm{dMMR}$ CRC related to LS versus sporadic $M L H 1-\mathrm{hm}$.

\section{Patients}

\section{MATERIALS AND METHODS}

Consecutive patients with CRC who had dMMR on IHC performed from May 1998 to May 2012 were included in the study. This consisted of patients enrolled in the Columbus LS study, ${ }^{2}$ and also included all patients with CRC diagnosed after 2006. Patients with $M L H 1$-hm tumors were identified by either no MLH1 protein on IHC and BRAF mutation or MLH1-hm. Patients classified as having LS had confirmed germ-line MMR mutations. Patients with dMMR tumors who did not have conclusive testing (i.e., not diagnosed as either LS or an MLH1-hm tumor) were excluded from further analysis.

Recurrent disease was defined as a recurrent tumor at the anastomotic site or distant metastasis that developed within 5 years of a primary diagnosis at stage I-III. Patients with less than 2 years of follow-up were excluded from this analysis. Synchronous tumors were defined as two colorectal tumors that were discovered simultaneously or within 6 months of each other. Metachronous colorectal tumors were discovered more than 6 months apart. ${ }^{16}$

Baseline information on demographics, tumor characteristics, treatment, and survival were obtained from medical charts. All tumors were pathologically reported according to the American Joint Committee on Cancer, 7th edition, for CRC. ${ }^{17}$ For patients with synchronous tumors, the tumor with the higher stage was documented as the primary tumor. The institutional review board at the Ohio State University approved this study.

\section{MSI, IHC, and MLH1 hypermethylation testing}

For patients in the Columbus LS study, DNA was extracted from paraffin-embedded tumor, normal adjacent tissue, and blood. IHC for the four MMR proteins, MSI testing, MLH1-hm testing, and germ-line genetic testing (sequencing and multiplex ligation probe assay) for the four MMR genes was performed as previously described. ${ }^{2,18-20}$ Tumor tissue used IHC to stain for MLH1 (Novacastra, Buffalo Grove, IL; NCL-L-MLH-1), MSH2 (Calbiochem (Merck Biosciences AG), Basel-Land, Switzerland; NA27), MSH6 (Epitomics, Burlingame, CA; AC-0047), and PMS2 proteins (BD Pharmingen, San Jose, CA; 556415). For patients enrolled in the Columbus LS study, the promoter region of $M L H 1$ was assessed for methylation with methylation-specific polymerase chain reaction. ${ }^{21}$ In clinical cases since 2005, DNA was modified with sodium bisulfite, and the bisulfite-treated DNA was sequenced by PyroMark MD (Qiagen, Hilden, Germany) for MLH1 methylation analysis. Exon 15 of the BRAF gene was sequenced in some clinical cases to identify any activating mutations. Approximately $25-50 \mathrm{ng}$ of tumor DNA was amplified in a $15-\mu \mathrm{l}$ polymerase chain reaction using Promega's GoTaq master mix (Promega, Madison, WI). Polymerase chain reaction products were analyzed using an ABI3700 sequencer (Life Technologies, Grand Island, NY) following suitable amplification.

\section{Survival analysis}

Age at diagnosis was defined as the age when a CRC diagnosis was confirmed. Overall survival (OS) was defined as the time from diagnosis to death from any cause. Patients who developed a metachronous primary CRC were censored at the time of diagnosis of the second tumor. Patients who were alive were censored at their last follow-up appointment date. Cancer-specific survival (CSS) was defined as the time from diagnosis to a CRC-related death, and we chose to censor patients who developed a second cancer (except for nonmelanoma skin cancer), died from other causes, or were lost to follow-up.

\section{Statistical analysis}

Descriptive statistics (median with quartiles for age, follow-up time, and carcinoembryonic level mean with standard deviation for other continuous variables; and frequency for categorical variables) were provided to summarize the patient population. Student's $t$-test and $\chi^{2}$ tests were used to compare the difference of continuous and categorical parameters in LS versus MLH1-hm groups, respectively. Kaplan-Meier estimation and the log-rank test were used to compare the difference of OS and CSS between the two groups. Stage of cancer and age at diagnosis were considered as covariates in the Cox proportional hazards model in the survival analysis. All statistical analyses were performed using SAS software version 9.4 (SAS Institute, Cary, NC).

\section{RESULTS}

Patient characteristics, stage at diagnosis, and location of tumor

A total of 189 patients with CRC and MMR deficiency documented by IHC were identified for the study period. Full testing (IHC, MLH1-hm, or BRAF mutation testing and germ-line sequencing) with conclusive results was obtained in 143 patients. MLH1-hm tumors were found in 106 patients $(74.1 \%$ of the cohort), and a germ-line MMR mutation was found in 37 
Table 1 Patient characteristics, tumor stage and location

\begin{tabular}{|c|c|c|c|}
\hline & $\begin{array}{l}\text { Lynch syndrome } \\
\quad(n=37)\end{array}$ & $\begin{array}{c}\text { MLH1-hm } \\
(n=106)\end{array}$ & $P$ value \\
\hline $\begin{array}{l}\text { Age, median years } \\
\text { (Q1, Q3) }\end{array}$ & $47(35,58)$ & $70.5(63,80)$ & $<0.0001$ \\
\hline \multicolumn{4}{|l|}{ Sex } \\
\hline Male & $22(59.5 \%)$ & $45(42.5 \%)$ & 0.074 \\
\hline Female & $15(40.5 \%)$ & $61(57.5 \%)$ & \\
\hline \multicolumn{4}{|l|}{ Race } \\
\hline Caucasian & $33(89.2 \%)$ & $88(89.8 \%)$ & 0.775 \\
\hline African American & $3(8.1 \%)$ & $9(9.2 \%)$ & \\
\hline Hispanic & $1(2.7 \%)$ & $1(1.0 \%)$ & \\
\hline \multicolumn{4}{|l|}{ Stage } \\
\hline I & $4(10.8 \%)$ & $15(14.2 \%)$ & 0.022 \\
\hline$\|$ & $10(27.0 \%)$ & $48(45.3 \%)$ & \\
\hline III & $17(46.0 \%)$ & $28(26.4 \%)$ & \\
\hline IV & $3(8.1 \%)$ & $14(13.2 \%)$ & \\
\hline I-III (unknown) & $3(8.1 \%)$ & $1(0.9 \%)$ & \\
\hline \multicolumn{4}{|l|}{ Location } \\
\hline Right & $27(73.0 \%)$ & $87(82.9 \%)$ & 0.457 \\
\hline Left & $7(18.9 \%)$ & $11(10.5 \%)$ & \\
\hline Rectum & $3(8.1 \%)$ & $7(6.7 \%)$ & \\
\hline Synchronous & $4(10.8 \%)$ & $6(5.7 \%)$ & 0.459 \\
\hline Metachronous & $7(18.9 \%)$ & $4(3.8 \%)$ & 0.009 \\
\hline \multicolumn{4}{|c|}{ Received adjuvant chemotherapy } \\
\hline Stage II & $3(30 \%)$ & $4(8.3 \%)$ & 0.058 \\
\hline Stage III & $10(59 \%)$ & $11(39 \%)$ & 0.091 \\
\hline Recurrence (\%) & $9(24.3 \%)$ & $12(11.3 \%)$ & 0.040 \\
\hline $\begin{array}{l}\text { Other cancer } \\
\text { diagnosis (\%) }\end{array}$ & $13(34.2 \%)$ & $27(25.5 \%)$ & 0.301 \\
\hline $\begin{array}{l}\text { Follow-up time, median } \\
\text { months (Q1, Q3) }\end{array}$ & $30.0(16,117)$ & $25(5,54)$ & 0.02 \\
\hline $\begin{array}{l}\text { Carcinoembryonic } \\
\text { antigen level, median } \\
n(\mathrm{Q} 1, \mathrm{Q} 3)\end{array}$ & $1.2(0.7,2.6)$ & $1.9(1.0,5.6)$ & 0.070 \\
\hline
\end{tabular}

Statistical significance was evaluated by the $\chi^{2}$ test for categorical variables and Student's $t$-test for continuous variables. Significance was set at $P<0.05$. MLH1-hm, sporadic inactivation of $M L H 1$ by hypermethylation; Q, quartile.

patients (25.9\% of the cohort). In the other 46 patients with dMMR tumors, further testing was either not feasible or was negative; these patients were excluded from further analysis. Patient characteristics are described in Table 1. Forty-eight patients were enrolled in the Columbus LS study (33.6\%), and 95 patients $(66.4 \%)$ were found on routine clinical screening; there were no statistically significant differences in the characteristics of the two patient populations. Of the 106 patients with MLH1-hm tumors, 25 patients had confirmed BRAF mutation analysis and the remainder had MLH1 hypermethylation testing. Patients with LS-associated tumors were significantly younger (median age, 47 vs. 70.5 years at diagnosis; $P<0.0001$ )
Table 2 Tumor characteristics and type of surgery

\begin{tabular}{|c|c|c|c|}
\hline & $\begin{array}{l}\text { Lynch syndrome } \\
\quad(n=37)\end{array}$ & $\begin{array}{c}\text { MLH1-hm } \\
(n=106)\end{array}$ & $P$ value \\
\hline \multicolumn{4}{|l|}{ Tumor type } \\
\hline Adenocarcinoma & $27(77.1 \%)$ & $78(74.3 \%)$ & \\
\hline Mucinous (>50\%) & $5(14.3 \%)$ & $21(20.0 \%)$ & 0.473 \\
\hline Signet ring & $3(8.6 \%)$ & $3(2.9 \%)$ & \\
\hline Medullary & $0(0 \%)$ & $2(1.9 \%)$ & \\
\hline Undifferentiated & $0(0 \%)$ & $1(1.0 \%)$ & \\
\hline \multicolumn{4}{|l|}{ Grade } \\
\hline Well differentiated & $2(5.9 \%)$ & $4(3.9 \%)$ & 0.641 \\
\hline Moderately differentiated & $21(61.8 \%)$ & $53(51.5 \%)$ & \\
\hline Poorly differentiated & $11(32.3 \%)$ & $45(43.7 \%)$ & \\
\hline Undifferentiated & $0(0 \%)$ & $1(1.0 \%)$ & \\
\hline \multicolumn{4}{|l|}{ Border } \\
\hline Infiltrative & $22(91.7 \%)$ & $66(89.2 \%)$ & \\
\hline Pushing & $0(0 \%)$ & $5(6.7 \%)$ & 0.32 \\
\hline Focally both & $2(8.3 \%)$ & $3(4.1 \%)$ & \\
\hline $\begin{array}{l}\text { Lymph nodes examined, } \\
\text { mean (SD) }\end{array}$ & $24.8(16.0)$ & $22.3(17.5)$ & 0.436 \\
\hline $\begin{array}{l}\text { Lymph nodes positive, } \\
\text { mean (SD) }\end{array}$ & $2.1(3.7)$ & $1.8(3.9)$ & 0.619 \\
\hline $\begin{array}{l}\text { Size (largest tumor), } \\
\text { mean (SD) }\end{array}$ & $5.8(3.2)$ & $5.9(2.6)$ & 0.922 \\
\hline \multicolumn{4}{|l|}{ Surgery } \\
\hline Right hemicolectomy & $16(44.4 \%)$ & $71(72.5 \%)$ & \\
\hline Left hemicolectomy & $6(16.7 \%)$ & $11(11.2 \%)$ & 0.0047 \\
\hline Subtotal colectomy & $2(5.6 \%)$ & $8(8.2 \%)$ & \\
\hline Total colectomy & $10(27.8 \%)$ & $5(5.1 \%)$ & \\
\hline LAR & $2(5.5 \%)$ & $3(3.0 \%)$ & \\
\hline
\end{tabular}

Statistical significance was evaluated by $\mathrm{Chi}$-square for categorical variables and $t$-test for continuous variables. Significance was set at $P<0.05$. The values given in mean (SD) are measured in centimeters.

LAR, low anterior resection; MLH1-hm, sporadic inactivation of $M L H 1$ by hypermethylation; SD, standard deviation.

and predominantly male ( 59.5 vs. $42.5 \% ; P=0.077$ ) compared with MLH1-hm patients. Most patients (72.9 and $71.7 \%$ in the LS and MLH1-hm groups, respectively) were diagnosed at stage II or III, and only 8.2 and $13.2 \%$ were diagnosed at stage IV in the two groups, respectively. Patients with LS were diagnosed with stage III disease significantly more often than patients with MLH1-hm tumors ( 46.0 vs. $26.4 \% ; P=0.022$ ), with a higher likelihood of recurrence ( 24.3 vs. $11.3 \%$; $P=0.040$ ). The division by stage and germ-line mutation was as follows: For MLH1 mutations, stages I-IV at diagnoses were $0,27.3,54.5$, and $9.1 \%$; for MSH2 mutations, 5.9, 17.6, 52.9, and 11.8\%; for MSH6 mutations, 40.0, 60.0, 0, and 0\%; and for PMS2 mutations, 0 , $33.3,66.7$, and $0 \%$. Table 1 shows the number of patients with LS and MLH1-hm tumors receiving 5-fluorouracil-based adjuvant chemotherapy in stage II and III disease, respectively. The median duration of follow-up was longer for patients with LS (27.5 vs. 25.0 months; $P=0.032$ ). 


\section{Tumor pathology and type of surgery}

Table 2 describes tumor pathology and type of surgery performed in the two groups. There were no differences in any of the pathology factors between the two groups. Patients with LS had total colectomies performed significantly more

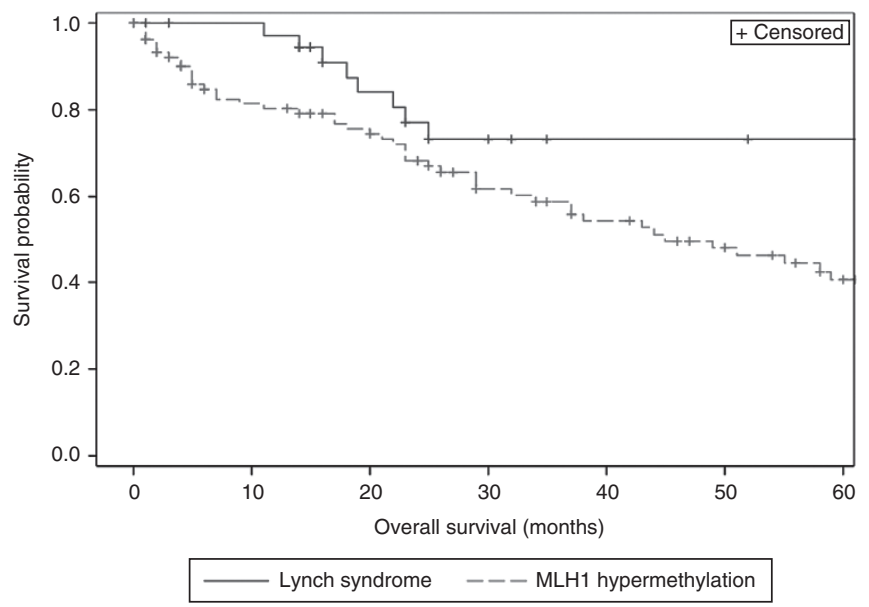

Figure 1 Overall survival (months) in all stages in patients with Lynch syndrome-associated colorectal cancer (CRC) and sporadic inactivation of MLH1 by hypermethylation CRC.

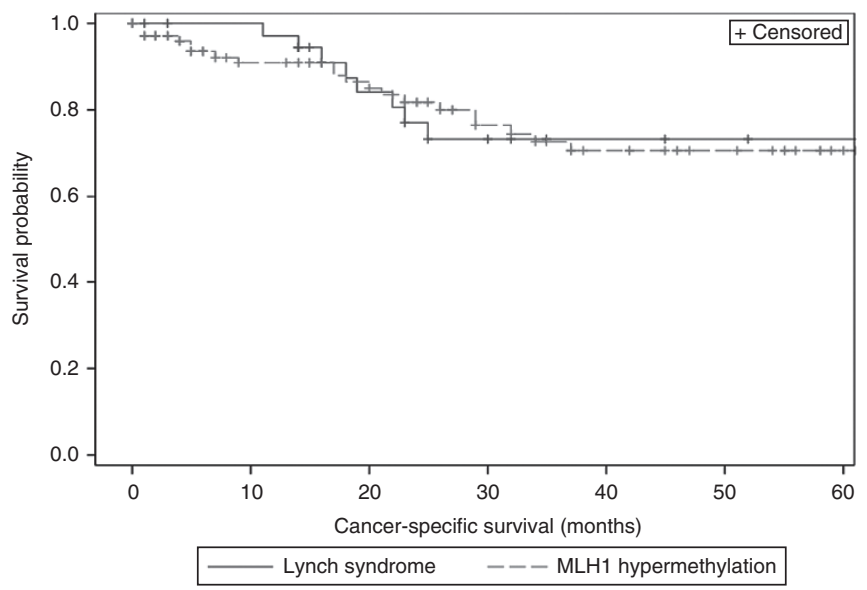

Figure 2 Cancer-specific survival (months) in all stages in patients with Lynch syndrome-associated colorectal cancer (CRC) and sporadic inactivation of MLH1 by hypermethylation CRC. often than patients with $M L H 1$-hm CRC (27.8 vs. 5.1\%; $P=0.0047)$. There were no statistically significant differences in the locations of the tumors; the majority were found in the right colon ( 73.0 and $82.1 \%$ in the two groups; $P=0.51$ ).

\section{Survival}

OS and CSS in the two groups are presented in Figures 1 and 2 and Table 3. Supplementary Figures S1-S3 online show CSS in the two groups broken down by stage at diagnosis. CSS events were not observed in patients with stage I disease and are not depicted in a figure. Table 3 presents median survival and hazard ratios for OS and CSS by stage and overall while adjusting for age and stage at diagnosis. The difference in CSS between the two groups was not statistically significant (hazard ratio $(\mathrm{HR})=1.33 ; P=0.60$ ) after adjusting for the effect of age and stage. Most CSS events occurred in the first 24 months after CRC diagnosis, and there was no significant difference between the groups when broken down by stage. OS was significantly longer for patients with LS and remained borderline significant after adjusting for the effect of age and stage $(\mathrm{HR}=1.96 ; P=0.095)$. This was mostly driven by the survival difference seen in stage II when broken down by stage $(\mathrm{HR}=3.97 ; P=0.079)$. No difference in OS and CSS was found when the LS group was limited to comparing patients with MLH1 germ-line mutations $(n=11)$ to patients with MLH1-hm tumors $(n=106)$ (calculations not shown). Patients with stage II or III cancer who received adjuvant chemotherapy had improved CSS, although this was not statistically significant $(\mathrm{HR}=0.48 ; P=0.25)$, compared with those without chemotherapy when including stage and age as covariates. There were better but not significant differences in CSS when comparing the LS group that received adjuvant chemotherapy with the MLH1-hm group receiving adjuvant chemotherapy after adjusting for age and stage ( $\mathrm{HR}=2.72 ; 95 \%$ confidence interval $=0.32-23.2 ; P=0.36$ ). There was no significant difference in CSS when comparing patients diagnosed before or after 2007 (HR $=0.90 ; P=0.81)$, and the results of patients with LS versus $M L H 1-\mathrm{hm}$ tumors were similar (HR $=1.38 ; P=0.57$ for CSS) after adjusting for time of diagnosis. Supplementary Table S1 online describes the germ-line mutations in the LS group.

Table 3 Median overall survival, cancer-specific survival, and hazard ratios corrected for age and stage at diagnosis

\begin{tabular}{|c|c|c|c|c|c|c|c|c|}
\hline & \multicolumn{2}{|c|}{$\begin{array}{l}\text { Overall survival } \\
\text { (months) }\end{array}$} & \multirow[b]{2}{*}{ HR $(95 \% \mathrm{Cl})$} & \multirow[b]{2}{*}{$P$ value } & \multicolumn{2}{|c|}{$\begin{array}{c}\text { Cancer-specific survival } \\
\text { (months) }\end{array}$} & \multirow[b]{2}{*}{ HR $(95 \% \mathrm{Cl})$} & \multirow[b]{2}{*}{$P$ value } \\
\hline & LS & MLH1-hm & & & LS & MLH1-hm & & \\
\hline Stage I & NR & 51 & NS & NS & NR & NR & NS & NS \\
\hline Stage II & $N R$ & 59 & $3.97(0.85-18.5)$ & 0.079 & NR & NR & $1.07(0.08-13.6)$ & 0.96 \\
\hline Stage III & 71 & 32 & $1.08(0.28-4.18)$ & 0.91 & 71 & NR & $1.11(0.22-5.64)$ & 0.90 \\
\hline Stage IV & 23.5 & 17 & $2.07(0.32-13.5)$ & 0.45 & 23.5 & 17 & $2.07(0.32-13.5)$ & 0.45 \\
\hline Overall & $N R$ & 45 & $1.96(0.89-4.92)$ & 0.095 & $N R$ & NR & $1.33(0.46-3.83)$ & 0.60 \\
\hline
\end{tabular}

$\mathrm{HR}>1$ signifies that LS has better survival than MLH1-hm patients.

$\mathrm{Cl}$, confidence interval; HR, hazard ratio; LS, Lynch syndrome; MLH1-hm, sporadic inactivation of MLH1 by hypermethylation; NR, not reached; NS, not enough events to calculate. 


\section{DISCUSSION}

In this study CSS is similar among patients with LS-associated CRC and sporadic MLH1-hm CRC. The lack of difference in CSS suggests that the prognosis for CRC is similar in patients with dMMR tumors, whether it is related to germ-line MMR mutations or sporadic MLH1 inactivation. OS was longer in patients with LS, as shown in Figure 1, but after correcting for age and stage at diagnosis the difference was of borderline significance and seems to be driven by patients with stage II disease. Both LS and MLH1-hm patients had exceptionally good survival for stage I (5-year CSS, 100\%) and stage II disease (5-year CSS, >90\%); this is better than expected for CRC with proficient MMR activity, as has been previously reported. ${ }^{9}$ Five-year CSS in stage III was $60 \%$ in both groups and similar to prognosis in MMR-proficient tumors. Benatti et al. ${ }^{22}$ looked at the prognostic impact of CRC with high MSI and the efficacy of chemotherapy in a cohort of 1263 patients with CRC. In a subanalysis, patients with germ-line MMR mutations had better CSS compared with patients with sporadic high MSI, but this impact disappeared in a multivariate analysis where only age and stage at diagnosis predicted CSS. Sinicrope et al. ${ }^{23}$ looked at germ-line MMR mutations versus MLH1-hm tumors in a study investigating the benefit of adjuvant 5-fluorouracil chemotherapy. Patients with dMMR tumors suspected to have germ-line mutations (based on clinical and IHC criteria) had improved survival compared with patients with sporadic dMMR tumors, but this association was lost after adjusting for age. Although more patients with LS received adjuvant therapy in our study, CSS was not significantly different between the two groups according to whether they received adjuvant chemotherapy. The interpretation of this analysis is limited because of the small sample size. Not surprisingly, patients with $M L H 1$-hm tumors tend to be older at diagnosis and more often female. Methylation and inactivation of genes is believed to be part of normal aging, but it is unclear why these tumors occur more frequently in females.

LS-associated tumors were more frequently diagnosed at stage III compared with $M L H 1$-hm tumors. This could explain the higher recurrence rates in the group with LS-associated CRC. It is possible that higher stage at presentation in younger patients with CRC is related to a lack of awareness of this disease among the younger population and lower rates of routine screening colonoscopies. It is possible that some patients with LS were diagnosed before entering this study, but many were diagnosed upon their CRC diagnosis and had no knowledge of the germ-line mutation.

There was no observed difference in pathologic features between the two groups in our study. Hartman et al. ${ }^{24}$ analyzed grade, histology, and tumor location in sporadic tumors with high MSI versus LS-associated/probable LS-associated tumors (based on BRAFV600E mutation, $M L H 1$-hm, cancer history, and germ-line MMR mutations). They found leftsided tumors with high MSI to be more frequently associated with LS. Sporadic tumors with high MSI demonstrated tumor-infiltrating lymphocytes more often (81 vs. 61\%) compared with LS-associated tumors, with other pathologic factors being similar.

In concordance with other studies, very few patients presented with stage IV disease in the two groups. The median OS in stage IV was similar to what would be expected for proficient MMR tumors (23.5 months) in the LS group but was worse in the MLH1-hm group (17 months). After adjusting for age, there was no significant difference between the two groups ( $\mathrm{HR}=1.96 ; 95 \% \mathrm{CI}=0.89-4.29)$. The interpretation of this is limited because of low patient numbers. Venderbosch et al. ${ }^{25}$ published survival data on AMMR CRC combining the CAIRO, CAIRO2, COIN, and FOCUS clinical trial data sets including patients with stage IV disease. They found that patients with dMMR tumors had poorer OS than patients with proficient MMR tumors (13.6 vs. 16.8 months; $\mathrm{HR}=1.35$; $95 \% \mathrm{CI}=1.13$ 1.61). The main cause of dMMR in the studies was $M L H 1-\mathrm{hm}$ (30 of 45 patients), and $73 \%$ of them had BRAF mutations. ${ }^{15}$

Our study has some limitations, including its retrospective nature and the relatively short median duration of follow-up (2 years). Also, the relatively small number of patients gives us limited power to detect small differences in CSS between the two groups, particularly when measuring the effect of adjuvant chemotherapy. However, one of the strengths of our study is that it was limited to patients with either LS or MLH1-hm tumors only by definitive molecular diagnosis.

In conclusion, we have shown that there is no statistically significant difference in CSS between LS-associated and MLH1-hm CRC in this cohort of patients. The observed difference in median OS became nonsignificant after correcting for age and stage at diagnosis, and is therefore likely confounded by the differences in median age and disease stages between the two groups.

\section{SUPPLEMENTARY MATERIAL}

Supplementary material is linked to the online version of the paper at http://www.nature.com/gim

\section{ACKNOWLEDGMENTS}

This study was supported by grants from the Ohio State University Comprehensive Cancer Center Core (grant CA16058) and the National Cancer Institute (CA67941).

\section{DISCLOSURE}

The authors declare no conflict of interest.

\section{REFERENCES}

1. Parsons R, Li GM, Longley MJ, et al. Hypermutability and mismatch repair deficiency in RER+ tumor cells. Cell 1993;75:1227-1236.

2. Hampel H, Frankel WL, Martin E, et al. Screening for the Lynch syndrome (hereditary nonpolyposis colorectal cancer). N Engl J Med 2005;352: 1851-1860.

3. Ligtenberg MJ, Kuiper RP, Chan TL, et al. Heritable somatic methylation and inactivation of MSH2 in families with Lynch syndrome due to deletion of the 3' exons of TACSTD1. Nat Genet 2009;41:112-117

4. Bandipalliam P. Syndrome of early onset colon cancers, hematologic malignancies \& features of neurofibromatosis in HNPCC families with homozygous mismatch repair gene mutations. Fam Cancer 2005;4:323-333. 


\section{ORIGINAL RESEARCH ARTICLE}

5. Mensenkamp AR, Vogelaar IP, van Zelst-Stams WA, et al. Somatic mutations in MLH1 and MSH2 are a frequent cause of mismatch-repair deficiency in Lynch syndrome-like tumors. Gastroenterology 2014;146:643-646.e8.

6. Haraldsdottir S, Hampel H, Tomsic J, et al. Colon and endometrial cancers with mismatch repair deficiency can arise from somatic, rather than germline, mutations. Gastroenterology 2014;147:1308-1316.e1.

7. Kim H, Jen J, Vogelstein B, Hamilton SR. Clinical and pathological characteristics of sporadic colorectal carcinomas with DNA replication errors in microsatellite sequences. Am J Pathol 1994;145:148-156.

8. Samowitz WS, Curtin K, Ma KN, et al. Microsatellite instability in sporadic colon cancer is associated with an improved prognosis at the population level. Cancer Epidemiol Biomarkers Prev 2001;10:917-923.

9. Popat S, Hubner R, Houlston RS. Systematic review of microsatellite instability and colorectal cancer prognosis. J Clin Oncol 2005;23:609-618.

10. Gryfe R, Gallinger S. Microsatellite instability, mismatch repair deficiency, and colorectal cancer. Surgery 2001;130:17-20.

11. Poynter JN, Siegmund KD, Weisenberger DJ, et al.; Colon Cancer Family Registry Investigators. Molecular characterization of MSI-H colorectal cancer by MLHI promoter methylation, immunohistochemistry, and mismatch repair germline mutation screening. Cancer Epidemiol Biomarkers Prev 2008;17:3208-3215.

12. Palomaki GE, McClain MR, Melillo S, Hampel HL, Thibodeau SN. EGAPP supplementary evidence review: DNA testing strategies aimed at reducing morbidity and mortality from Lynch syndrome. Genet Med 2009;11:42-65.

13. Parsons MT, Buchanan DD, Thompson B, Young JP, Spurdle AB. Correlation of tumour BRAF mutations and MLH1 methylation with germline mismatch repair (MMR) gene mutation status: a literature review assessing utility of tumour features for MMR variant classification. J Med Genet 2012;49:151-157.

14. Network NCC. NCCN Genetic/Familial High-Risk Assessment: Colorectal. Version 1.2014. 2014

15. Giardiello FM, Allen JI, Axilbund JE, et al.; US Multi-Society Task Force on Colorectal Cancer. Guidelines on genetic evaluation and management of
Lynch syndrome: a consensus statement by the US Multi-Society Task Force on colorectal cancer. Gastroenterology 2014;147:502-526.

16. Moertel CG. Multiple primary malignant neoplasms: historical perspectives. Cancer 1977;40(4 suppl):1786-1792.

17. Edge S, Byrd D, Compton CC, Fritz A, Greene F, Trotti A. AJCC Cancer Staging Manual . 7th edn. New York: Springer; 2010

18. Hampel H, Frankel W, Panescu J, et al. Screening for Lynch syndrome (hereditary nonpolyposis colorectal cancer) among endometrial cancer patients. Cancer Res 2006;66:7810-7817

19. Hampel H, Panescu J, Lockman J, et al. Comment on: screening for Lynch syndrome (hereditary nonpolyposis colorectal cancer) among endometrial cancer patients. Cancer Res 2007;67:9603.

20. Hampel H, Frankel WL, Martin E, et al. Feasibility of screening for Lynch syndrome among patients with colorectal cancer. J Clin Oncol 2008;26:57835788.

21. Herman JG, Umar A, Polyak K, et al. Incidence and functional consequences of hMLH1 promoter hypermethylation in colorectal carcinoma. Proc Natl Acad Sci USA 1998;95:6870-6875.

22. Benatti $P$, Gafà $R$, Barana $D$, et al. Microsatellite instability and colorectal cancer prognosis. Clin Cancer Res 2005;11:8332-8340.

23. Sinicrope FA, Foster NR, Thibodeau SN, et al. DNA mismatch repair status and colon cancer recurrence and survival in clinical trials of 5-fluorouracil-based adjuvant therapy. J Nat/ Cancer Inst 2011;103:863-875.

24. Hartman DJ, Brand RE, Hu H, et al. Lynch syndrome-associated colorectal carcinoma: frequent involvement of the left colon and rectum and lateonset presentation supports a universal screening approach. Hum Pathol 2013:44:2518-2528

25. Venderbosc h S, Nagtegaal ID, Maughan TS, et al. Mismatch repair status and BRAF mutation status in metastatic colorectal cancer patients: a pooled analysis of the CAIRO, CAIRO2, COIN, and FOCUS studies. Clin Can Res 2014;20:53225330. 